\title{
ADSORÇÃO DO CORANTE TÊXTIL REATIVO PRETO 5 DE SOLUÇÕES AQUOSAS UTILIZANDO QUITOSANA COM DIFERENTES GRAUS DE DESACETILAÇÃO NAS FORMAS DE PÓ E FILME
}

\author{
J. M. MOURA ${ }^{1}$, D. A. S. RODRIGUES ${ }^{1}$, D. D. R. GRÜNDMANN ${ }^{1}$, C. M. MOURA ${ }^{2}$ e L. A. A. \\ PINTO $^{1}$. \\ ${ }^{1}$ Universidade Federal do Rio Grande, Escola de Química e Alimentos \\ ${ }^{2}$ Universidade Federal do Pampa, Departamento de Engenharia de Alimentos
}

E-mail para contato: catarinamoura@unipampa.edu.br

\begin{abstract}
RESUMO - Nesta pesquisa, quitosana em pó (PQ) e filmes de quitosana (FQ) foram comparados como adsorventes para a remoção do corante têxtil reativo preto 5 (RP5) de soluções aquosas. PQ e FQ foram obtidas com diferentes graus de desacetilação (GD) (65, 75,85 e $95 \%$ ). O estudo de adsorção foi realizado, sob diversas condições experimentais, pelas isotermas de equilíbrio e termodinâmica. Verificou-se que PQ e FQ com GD de 95\% foram os adsorventes mais adequados, com taxas de remoção de RP5>99\% em pH 4,0.Para ambos, PQ e FQ, o modelo de Langmuir foi o mais adequado para representar os dados de equilíbrio. As capacidades máximas de adsorção foram 655 e $590 \mathrm{mgg}^{-1}$ para PQ e FQ, respectivamente, obtidos a $298 \mathrm{~K}$. O processo de adsorção foi espontâneo, favorável e exotérmico.
\end{abstract}

\section{INTRODUÇÃO}

As indústrias têxteis geram grandes quantidades de efluentes contendo corantes(Mezohegyiet al., 2012). O lançamento inadequado desses efluentes coloridos nos corpos de água pode causar danos graves do ponto de vista ecológico (Verma et al.,2012), sendo ambientalmente importante o seu tratamento (Salleh et al., 2011). Dentre os métodos utilizados para a remoção de corantes de águas residuais pode-se citar a adsorção(Demirbas, 2009). A quitosana tem sido considerada um adsorvente interessante, por pode ser obtida de fontes renováveis e proporcionar alta eficiência na remoção de corantes (Sreelatha et al., 2011).

Quanto ao uso de quitosana para remoção de corantes por adsorção, diferentes formas físicas têm sido empregadas, tais como, filmes, pó, fibras, hidrogéis entre outros (WanNgah et al., 2011). Estudos demonstraram que quitosana na forma de pó e de filmes são alternativas atrativas para remover corantes a partir de soluções aquosas (Rego et al., 2013; Dotto et al., 2013).

O objetivo deste trabalho foi a comparação entre a quitosana em pó (PQ) e filmes de quitosana 
(FQ) como adsorventes para a remoção do corante têxtil reativo preto 5 (RP5) de soluções aquosas.

\section{MATERIAIS E MÉTODOS}

\subsection{Características do corante}

O corante têxtil reativo preto 5

(RP5) (pureza de 95\%; peso molecular de $991,8 \mathrm{gmol}^{-1} ; \lambda_{\max }=596$ $\mathrm{nm}$, índice de cor de 20505) foi escolhido como por ser um representante dos azocorantes, aniônico e por ser extensivamente usado na indústria têxtil. Foi fornecido por Sigma-Aldrich (Brasil) e utilizado sem purificação adicional. Todas as soluções foram preparadas com água destilada.

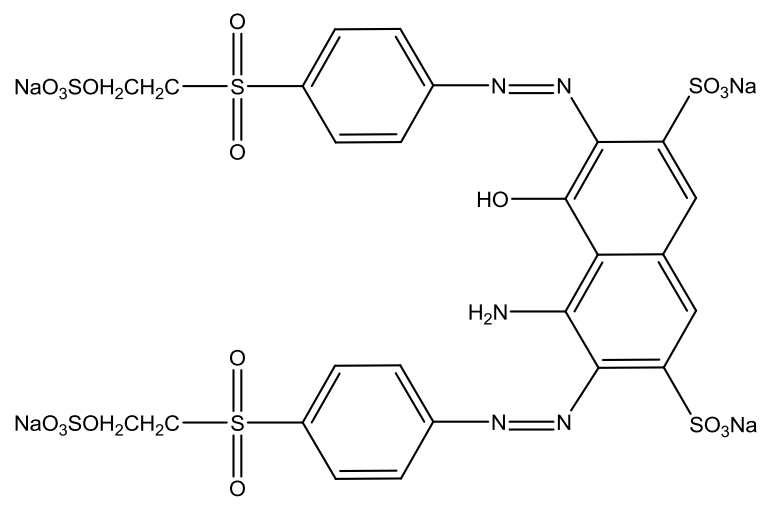

Figura 1 -Estrutura molecular do corante Reativo Preto 5 (RP5).

\subsection{Preparação e caracterização do pó e dos filmes de quitosana}

Resíduos de camarão (Penaeus brasiliensis) foram submetidos às etapas de desmineralização, desproteinização e desodorização (Weska et al., 2007) obtendo-se a quitina. A reação de desacetilação foi efetuada de acordo com Moura et al. (2011), a fim de obter quitosanas com graus de desacetilação (GD) de 65, 75, 85 e 95\%. As amostras de PQ (diâmetro médio $72 \pm 3 \mu \mathrm{m}$ ) foram caracterizadas quanto aos graus de desacetilação por titulação potenciométrica (Jiang et al., 2003), quanto as suas massas molares pelo método viscosimétrico (Zhang e Neau, 2001) e a área superficial específica pelo método BET (Cheung et al., 2009).

Os filmes de quitosana (FQ) foram obtidos utilizando cada uma das amostras de quitosana produzidas (GD de 65, 75, 85 e 95\%). O pó de quitosana foi dissolvido em solução de ácido acético 0,1 molL $^{-1}$ com agitação moderada [Marte, MAG-01H, Brasil], à temperatura ambiente. A solução foi centrifugada [Fanem, 206 BL, Brasil] e vertida sobre uma placa de plexiglas. Em seguida, o FQ foi obtido por evaporação do solvente e acondicionado em dessecador, atésua utilização (Dotto et al., 2013). Os FQ foram caracterizados quanto à resistência à tração e alongamento utilizando um

analisador de textura [Stable Micro Systems, TA-XT-2i, Reino Unido] de acordo com os procedimentos da ASTM (ASTM, 2001). A espessura foi obtida por um micrômetro digital [Insize, IP54, Brasil], com 0,0010 mm de resolução. Para os ensaios de adsorção os FQ foram divididos em porções de tamanho de $1 \mathrm{~cm}$ x $1 \mathrm{~cm}$.

\subsection{Experimentos de adsorção}

Os ensaios de adsorção foram realizados em batelada em um agitador tipo Wagner [Nova Ética, 
$218 \mathrm{MBD}$, Brasil]. Soluções de RP5 $\left(2,00 \mathrm{gL}^{-1}\right)$ foram preparadas e o seu $\mathrm{pH}$ foi ajustado $(4,0 ; 6,0$ e $8,0)$ com uma solução tampão de fosfato dissódico/ácido cítrico $0,1 \mathrm{~mol} \cdot \mathrm{L}^{-1}$. Os ensaios foram realizados em três etapas, com a dosagem de adsorvente de $500 \mathrm{mgL}^{-1}$ : na primeira etapa, foram avaliados o efeito do $\mathrm{pH}(4,0,6,0$ e 8,0), o grau de desacetilação $(65,75,85$ e 95\%) e tipo de adsorvente (PQ e FQ), com concentração inicial de corante $100 \mathrm{mgL}^{-1}$, a temperatura a $298 \mathrm{~K}$ e velocidade de agitação de $100 \mathrm{rpm}$, sendo as soluções agitadas até que o equilíbrio; na segunda etapa, foram obtidas as isotermas de equilíbrio para PQ e FQ nas condições mais adequadas de $\mathrm{pH}$ e grau de desacetilação. As soluções foram agitadas (100 rpm) até atingir o equilíbrio, a 298, 308, 318 e $328 \mathrm{~K}$. Para os testes acima, a concentração de RP5 foi determinada por espectrofotometria [Biospectro, SP22, Brasil]. A quantidade de RP5 adsorvido por grama de adsorvente no equilíbrio ( $\mathrm{q}_{\mathrm{e}}, \mathrm{mgg}^{-1}$ ), foram calculadas $\left(\mathrm{q}_{\mathrm{t}}, \mathrm{mgg}^{-1}\right)$ e o percentual de remoção $(\% \mathrm{R})$, conforme Equações 1, 2 e 3 respectivamente:

$$
\begin{aligned}
& q_{e}=\frac{C_{0}-C_{e}}{m} V \\
& q_{t}=\frac{C_{0}-C_{t}}{m} V \\
& \% R=\frac{C_{0}-C_{e}}{C_{0}} \times 100
\end{aligned}
$$

em que $C_{0}$ é a concentração inicial de corante $\left(\mathrm{mgL}^{-1}\right), C_{e}$ é a concentração de corante no equilíbrio $\left(\mathrm{mgL}^{-1}\right), C_{t}$ é a concentração de corante no tempo $\left(\mathrm{mgL}^{-1}\right), V$ é o volume da solução de corante utilizado (L), e $m$ é a massa do adsorvente utilizado (mg).

\subsection{Isotermas de Equilíbrio e Termodinâmica}

As curvas de equilíbrio foram obtidas a 298, 308, 318 e 328 K. Para obter informações sobre a adsorção do RP5 para PQ e FQ, os modelos de isotermas de Langmuir e de Freundlich, conforme Equações 4 e 5, respectivamente:

$$
\begin{aligned}
& q_{e}=\frac{q_{m} k_{L} C_{e}}{1+k_{L} C_{e}} \\
& q_{e}=k_{F} C_{e}^{1 / n}
\end{aligned}
$$

sendo $C_{e}$ a concentração de equilíbrio do corante em solução $\left(\mathrm{mgL}^{-1}\right), q_{e}$ a capacidade de adsorção no equilíbrio $\left(\mathrm{mgg}^{-1}\right), q_{m}$ a capacidade máxima de adsorção do adsorvente para completar a cobertura da monocamada $\left(\mathrm{mgg}^{-1}\right)$, e $k_{L}$ a constante de adsorção de Langmuir $\left(\mathrm{mgL}^{-1}\right), k_{F}$ e $1 / n_{F}$ constantes características que representam a capacidade e a intensidade de adsorção do sistema, respectivamente. 
O comportamento termodinâmico de adsorção foi avaliado pela estimativa da mudança da energia livre de Gibbs $\left(\Delta G^{0}, \mathrm{~kJ} \mathrm{~mol}^{-1}\right)$, variação de entalpia $\left(\Delta H^{0}, \mathrm{~kJ} \mathrm{~mol}^{-1}\right)$ e variação de entropia $\left(\Delta S^{0}\right.$, $\mathrm{kJ} \mathrm{mol}^{-1} \mathrm{~K}^{-1}$ ). Estes parâmetros foram estimados pelas Equações 6 e 7 (Liu, 2009):

$$
\begin{aligned}
& \Delta G^{0}=-R T \ln \left(\rho_{w} K_{D}\right) \\
& \ln \left(\rho_{w} K_{D}\right)=\frac{\Delta S^{0}}{R}-\frac{\Delta H^{0}}{R T}
\end{aligned}
$$

em que $K_{D}$ é a constante de equilíbrio termodinâmico $\left(\mathrm{Lmol}^{-1}\right)$.

O equilíbrio foi determinado por ajuste dos modelos (Equações 4 e 5)aos dados experimentais, por meio de regressão não linear, utilizando o método de estimação Quasi-Newton. Os cálculos foram realizados pelo software Statistica 7.0 (Statsoft, EUA). O ajuste da qualidade e da precisão dos parâmetros foi medido pelo coeficiente de determinação $\left(R^{2}\right)$ e erro relativo médio $(E M R)$.

\section{RESULTADOS E DISCUSSÃO}

\subsection{Características do PQ e do FQ}

As amostras de pó de quitosana apresentaram graus de desacetilação de $65 \pm 1 \%, 75 \pm 2 \%, 85 \pm$ $1 \%$ e $95 \pm 1 \%$. Para todas as amostras de quitosana em pó, os valores de massa molar variaram de 140 a $160 \mathrm{kDa}$, e a área superficial específica foi de $4,4 \pm 0,2 \mathrm{~m}^{2}$. Em relação aos filmes de quitosana, houve diminuição da resistência à tração em função do aumento do grau de desacetilação e os valores variaram de 23,8 $\pm 0,1$ a 39,2 $\pm 0,1 \mathrm{MPa}$. Tendência semelhante foi observada para o alongamento, sendo que os valores variaram de $9,5 \pm 0,1$ a $15,7 \pm 0,1 \%$, e a espessura de todas as amostras de filmesfoi de $62 \pm 4 \mathrm{~mm}$.

\subsection{Efeito do pH e do grau de desacetilação}

A Figura 2 apresenta os efeitos do $\mathrm{pH}(4,0 ; 6,0$ e 8,0) e do grau de desacetilação $(65 \%, 75 \%$, $85 \%$ e 95\%) sobre a adsorção do RP5 para PQ e FQ. Na Figura 2 (2a, 2b e 2c), verificou-se que a adsorção de RP5 foi favorecida pela diminuição do $\mathrm{pH}$ de 8,0 para 4,0, para PQ e FQ. Isto pode ter ocorrido, porque o aumento de íons $\mathrm{H}^{+}$presentes na solução com a diminuição do $\mathrm{pH}$ facilita a protonação dos grupos amino da quitosana $\left(\mathrm{NH}_{2}\right)$, os quais foram convertidos em $\mathrm{NH}_{3}{ }^{+}$. Em geral, para os filmes de quitosana (FQ) e pós de quitosana(PQ), o aumento do grau de desacetilação causou um aumento nos valores de $\mathrm{Q}$ e $\mathrm{R}$ (Figura2). Isto aconteceu porque quando o grau de desacetilação aumenta de $65 \%$ a $95 \%$, mais grupos $\mathrm{NH}_{2}$ estavam disponíveis para interagir com $\mathrm{RP} 5$, melhorando o desempenho da adsorção.

Comparando o PQ e FQ na Figura 2, pode-se observar que os PQ apresentaram valores de q e $\mathrm{R}$ superiores aos $\mathrm{FQ}$, na maioria das condições experimentais. Isto pode ser atribuído a maior área 
disponível (por unidade de massa) do PQ. No entanto, em pH 4,0 e grau de desacetilação de 95\% (Figura 2a), os valores de q e R do PQ e do FQ foram semelhantes. Isto pode ser explicado por que em baixos valores de $\mathrm{pH}$ e alto grau de desacetilação, a quitosana apresenta uma quantidade maior de amino grupos protonados que causam uma repulsão entre as cadeias poliméricas no FQ, melhorando os aspectos de transferência de massa (Rego et al., 2013; Dotto et al., 2013).
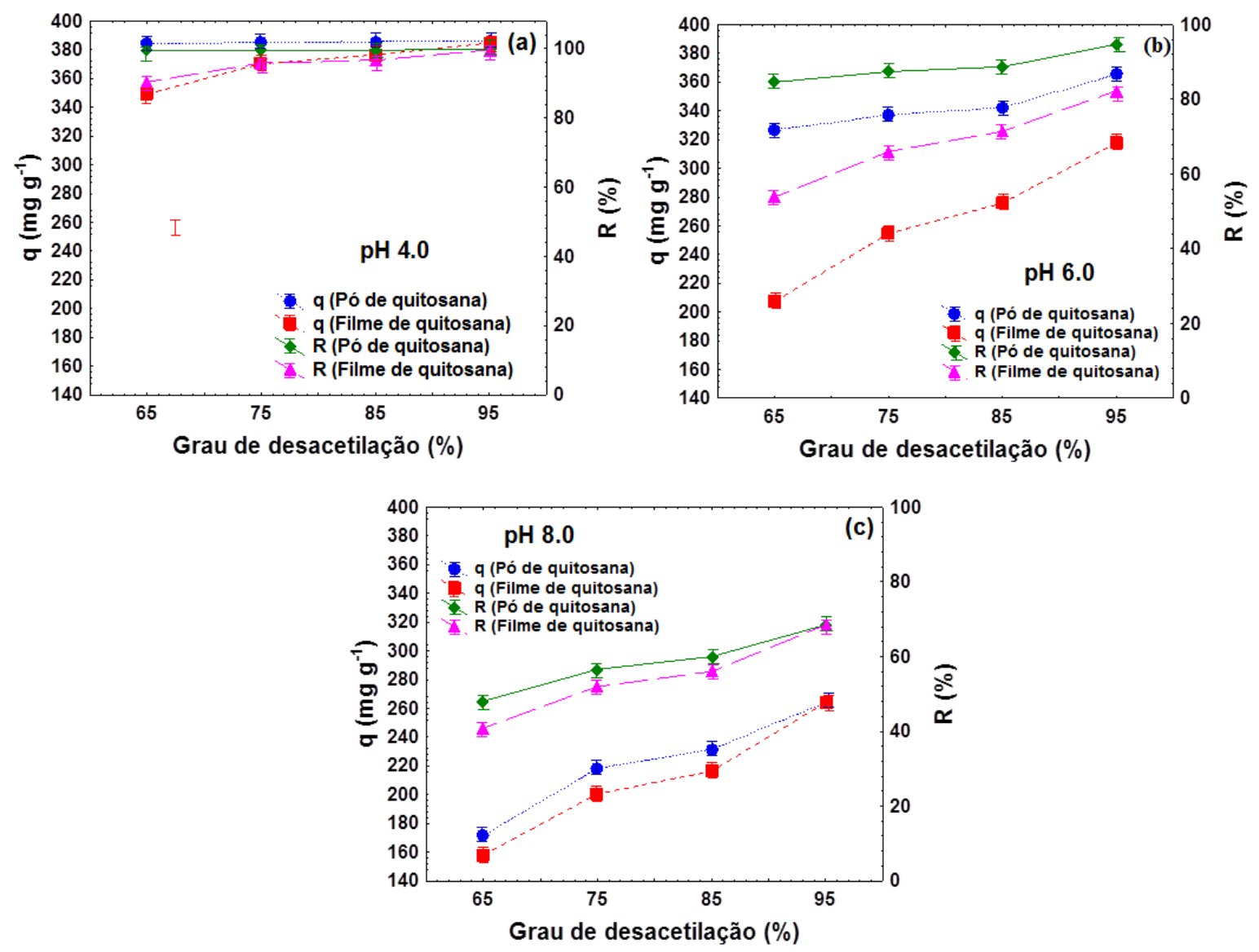

Figura 2-Efeito do grau de desacetilação na adsorção de RP5 para quitosana em pó e filmes de quitosana: (a) $\mathrm{pH} 4,0$, (b) $\mathrm{pH}$ 6,0 e (c) o pH 8,0.

\subsection{Isotermas de Equilíbrio e Termodinâmica}

As curvas de equilíbrio de adsorção foram obtidas a 298, 308, 318 e $328 \mathrm{~K}$, a fim de verificar o efeito da temperatura. Estas curvas são apresentadas na Figura 3, onde observa-se que para ambos, PQ (Figura 3a) e FQ (Figura 3b), as isotermas foram caracterizadas como tipo I (Blázquezet al., 2010), demonstrando que haviam numerosos sítios de adsorção de fácil acesso, e, também, que ocorreu a saturação na monocamada. Além disso, na Figura 3, pode-se observar que a capacidade de adsorção foi aumentada com a diminuição da temperatura, sendo que os valores máximos foram obtidos a 298 $\mathrm{K}$. Isto ocorreu porque o aumento da temperatura conduz a um aumento na solubilidade corantes, assim, as forças de interação entre corantes e solvente torna-se mais forte do que entre corantes e 
adsorvente (Crini e Badot, 2008). Os parâmetros de equilíbrio para a adsorção de RP5 por PQ e FQ utilizando os modelos de Langmuir (Equação 4) e Freundlich (Equação 5) estão na Tabela 1.
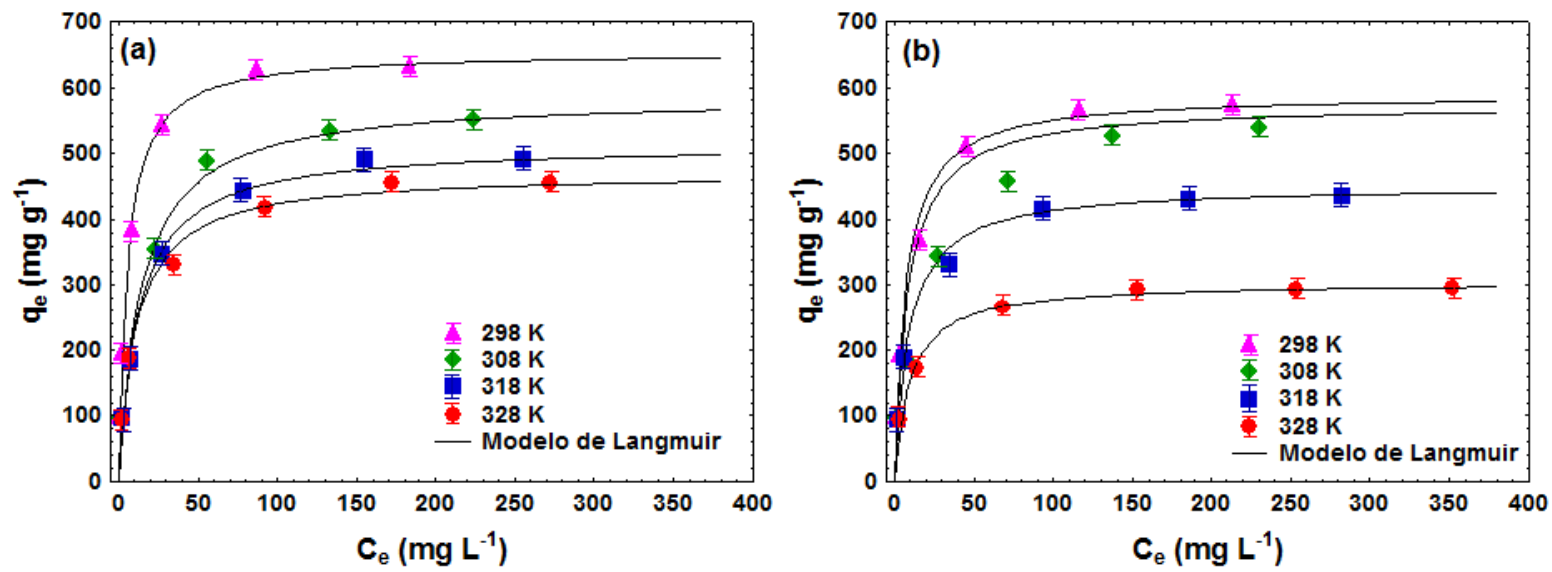

Figura 3 -Curvas de equilíbrio da adsorção do RP5: (a) pó de quitosana e (b) filme de quitosana $298 \mathrm{~K}$; $308 \mathrm{~K}$; $318 \mathrm{~K}$; $328 \mathrm{~K}$ ).

Tabela 1.Parâmetros de equilíbrio para a adsorção de RP5 sobre o pó e o filme de quitosana

\begin{tabular}{|c|c|c|c|c|c|}
\hline & & & K) & & \\
\hline & Langmuir & 298 & 308 & 318 & 328 \\
\hline & $k_{L}\left(\mathrm{Lmg}^{-1}\right)$ & 0,18 & 0,09 & 0,08 & 0,07 \\
\hline Quitosana em & $q_{m}\left(\mathrm{mgg}^{-1}\right)$ & 655 & 587 & 514 & 471 \\
\hline pó (PQ) & $R^{2}$ & 0,994 & 0,987 & 0,994 & 0,992 \\
\hline & $\operatorname{EMR}(\%)$ & 1,3 & 1,7 & 0,8 & 0,4 \\
\hline & $k_{L}\left(\mathrm{Lmg}^{-1}\right)$ & 0,14 & 0,12 & 0,11 & 0,1 \\
\hline Filme de & $q_{m}\left(\mathrm{mgg}^{-1}\right)$ & 590 & 574 & 451 & 305 \\
\hline (FQ) & $R^{2}$ & 0,986 & 0,985 & 0,996 & 0,995 \\
\hline & $\operatorname{EMR}(\%)$ & 1,6 & 1,9 & 0,3 & 0,4 \\
\hline & Freundlich & & & & \\
\hline & $k_{F}\left(\mathrm{mgg}^{-1}\right)\left(\mathrm{mgL}^{-1}\right)^{-1 / n} \mathrm{~F}$ & 204 & 135 & 125 & 117 \\
\hline Quitosana em & $n_{F}$ & 4,2 & 3,6 & 3,8 & 3,9 \\
\hline pó (PQ) & $R^{2}$ & 0,894 & 0,923 & 0,922 & 0,926 \\
\hline & $\operatorname{EMR}(\%)$ & 2,4 & 1,8 & 1,8 & 1,5 \\
\hline & $k_{F}\left(\mathrm{mgg}^{-1}\right)\left(\mathrm{mgL}^{-1}\right)^{-1 / \mathrm{n}}$ & 171 & 122 & 116 & 98 \\
\hline Filme de & $n_{F}$ & 4,1 & 3,4 & 4,1 & 4,9 \\
\hline quitosana(FQ) & $R^{2}$ & 0,916 & 0,952 & 0,903 & 0,901 \\
\hline & $\operatorname{EMR}(\%)$ & 3,9 & 3,5 & 7,6 & 8,3 \\
\hline
\end{tabular}


Os altos valores do coeficiente de determinação $\left(R^{2}>0,98\right)$ e os baixos valores do erro médio relativo $(E M R<2,0 \%)$ (Tabela 1) mostram que o modelo de Langmuir foi o mais adequado para representar a adsorção do RP5 para PQ e FQ. Os valores de $k_{L}$ (Tabela 1) aumentou com a diminuição da temperatura, que mostra que a afinidade adsorventes-RP5 foi máxima a $298 \mathrm{~K}$. A mesma tendência foi encontrada para $q_{m}$, mostrando que a capacidade de adsorção foi favorecida a $298 \mathrm{~K}$. A constante de equilíbrio termodinâmico $\left(K_{D}\right)$, a energia livre de Gibbs $(\Delta G)$, variação de entalpia $(\Delta H)$ e variação de entropia $(\Delta S)$ foram estimados, e os resultados são apresentados na Tabela 2.

Os valores de $K_{D}$ (Tabela 2) aumentaram com a diminuição da temperatura, confirmando que a adsorção de RP5 para PQ e FQ foi favorecida a $298 \mathrm{~K}$. Os valores negativos e elevados de $\Delta G$ indicam que a adsorção de um RP5 foi um processo espontâneo e energeticamente favorável. Os valores negativos de $\Delta H$ indicam um processo exotérmico. Além disso, a magnitude do $\Delta H$ é consistente com fisissorção devido às interações eletrostáticas. Os valores de $\Delta S$ foram negativos para o PQ e positiva para o FQ (Tabela 2). No entanto, observou-se que a variação de entalpia $(\Delta H)$ contribuiu mais do que a variação de entropia $(\Delta S)$ para obter os valores negativos de $\Delta G$ (de acordo com os valores de $(\Delta H)$ e $(T \Delta S)$ na Tabela 2. Isto mostra que a adsorção RP5 para PQ e FQ foi um processo controlado pela entalpia.

Tabela 2.Parâmetros termodinâmicos para a adsorção do RP5 sobre o pó e o filme de quitosana

\begin{tabular}{|c|c|c|c|c|c|c|}
\hline & $\begin{array}{c}T \\
(\mathrm{~K}) \\
\end{array}$ & $\begin{array}{c}K_{D} \\
\left(\mathrm{Lg}^{-1}\right)^{*}\end{array}$ & $\begin{array}{c}\Delta G^{0} \\
\left(\mathrm{~kJ} \mathrm{~mol}^{-1}\right)^{*}\end{array}$ & $\begin{array}{c}\Delta H^{0} \\
\left(\mathrm{~kJ} \mathrm{~mol}^{-1}\right)^{*}\end{array}$ & $\begin{array}{c}\Delta S^{0} \\
\left(\mathrm{~kJ} \mathrm{~mol}^{-1} \mathrm{~K}^{-1}\right)^{*}\end{array}$ & $\begin{array}{c}T \Delta S^{0} \\
\left(\mathrm{~kJ} \mathrm{~mol}^{-1}\right)^{*}\end{array}$ \\
\hline \multirow{4}{*}{$\begin{array}{c}\text { Pó de } \\
\text { quitosana } \\
\text { (PQ)* }\end{array}$} & 298 & $118,1 \pm 1,2$ & $-28,6 \pm 0,8$ & \multirow{4}{*}{$-33,7 \pm 1,3$} & \multirow{4}{*}{$-0,017 \pm 0,002$} & $-5,1 \pm 0,2$ \\
\hline & 308 & $53,1 \pm 0,8$ & $-28,4 \pm 0,8$ & & & $-5,3 \pm 0,3$ \\
\hline & 318 & $40,8 \pm 1,2$ & $-28,3 \pm 0,7$ & & & $-5,4 \pm 0,1$ \\
\hline & 328 & $33,2 \pm 1,1$ & $-28,1 \pm 0,7$ & & & $-5,6 \pm 0,1$ \\
\hline \multirow{4}{*}{$\begin{array}{l}\text { Filme de } \\
\text { quitosana } \\
\text { (FQ)* }\end{array}$} & 298 & $82,7 \pm 0,3$ & $-28,2 \pm 0,5$ & \multirow{4}{*}{$-27,0 \pm 0,9$} & \multirow{4}{*}{$0,004 \pm 0,002$} & $1,2 \pm 0,1$ \\
\hline & 308 & $69,1 \pm 0,6$ & $-28,3 \pm 0,5$ & & & $1,3 \pm 0,1$ \\
\hline & 318 & $49,1 \pm 1,2$ & $-28,4 \pm 0,5$ & & & $1,4 \pm 0,1$ \\
\hline & 328 & $30,2 \pm 0,5$ & $-28,3 \pm 0,5$ & & & $1,3 \pm 0,1$ \\
\hline
\end{tabular}

\section{CONCLUSÃO}

A quitosana em pó (PQ) e os filmes de quitosana (FQ) foram comparados como adsorventes para a remoção de reativo preto 5 (RP5) de soluções aquosas. Os resultados mostraram que o PQ e FQ, com grau de desacetilação de 95\%, a pH 4, removeram 99\% de RP5 da solução. O modelo de Langmuir foi adequado para representar os dados de equilíbrio, e as capacidades máximas de adsorção foram 655 e $590 \mathrm{mgg}^{-1}$ para PQ e FQ, respectivamente, a $298 \mathrm{~K}$. O processo de adsorção foi espontânea, favorável e exotérmica. PQ e FQ foram bons adsorventes para o RP5, onde PQ apresentou capacidade de adsorção ligeiramente maior. 


\section{9 a 22 de outubro de 2014 \\ Florianópolis/SC}

\section{REFERÊNCIAS}

BLÁZqueZ, G., CAlERO, M., HERnÁINZ, F., TENORIO, G., MARTíN-LARA, M. A. Equilibrium biosorption of lead (II) from aqueous solutions by solid waste fromolive-oil production.Chem. Eng. J.,v. 160, p. 615-622, 2010.

CRINI, G., BADOT, P.M. Application of chitosan, a natural aminopolysaccharide, for dye removal from aqueous solutions by adsorption processes using batch studies: A review of recent literature. Prog. Polym. Sci.,v. 33, p. 399-447, 2008.

CHEUNG, W.H., SZETO, Y.S., MCKAY, G. Enhancing the adsorption capacities of acid dyes by chitosan nano particles. Bioresource Technol., v. 100, p. 1143-1148, 2009.

DOTTO, G.L., PINTO, L.A.A. Adsorption of food dyes acid blue 9 and food yellow 3onto chitosan: Stirring rate effect in kinetics and mechanism. J.Hazard. Mater.,v. 187, p. 164-170, 2011.

DOTTO, G.L., MOURA, J.M., CADAVAL JR., T.R.S., PINTO, L.A.A. Application of chitosan films for the removal of food dyes from aqueous solutions by adsorption. Chem. Eng. J.,v. 214, p. 8 16, 2013.

JIANG, X., CHEN, L., ZHONG, W.I. A new linear potentiometric titration method for the determination of deacetylation degree of chitosan. Carbohydr. Polym.,v. 54, p. 457-463, 2003.

LIU, Y.Is the free energy change of adsorption correctly calculated.J. Chem. Eng. Data.,v. 54, p. 1981-1985, 2009.

MEZOHEGYI, G., VAN DER ZEE, F.P., FONT, J., FORTUNY, J., FABREGAT, A. Towards advanced aqueous dye removal processes: A short review on the versatile role of activated carbon. J. Environ. Manage., v. 102, p. 148-164, 2012.

MOURA, C.M., MOURA, J.M., SOARES, N.M., PINTO, L.A.A. Evaluation of molar weight and deacetylation degree of chitosan during chitin deacetylation reaction: used to produce biofilm. Chem. Eng. Process.,v. 50, p. 351-355, 2011.

RÊGO, T.V., CADAVAL JR., T.R.S., DOTTO, G.L., PINTO, L.A.A.Statistical optimization, interaction analysis and desorption studies for the azo dyes adsorption onto chitosan films. J. Colloid Interface Sci.,v. 411, p. 27-33, 2013.

SALLEH, M.A.M., MAHMOUD, D.K., KARIM, W.A.W.A. IDRIS,. Cationic and anionic dye adsorption by agricultural solid wastes: A comprehensive review. Desalination,v. 280, p. 1-13, 2011.

SREElATHA, G., AGEETHA, V., PARMAR, J., PADMAJA, P.Equilibrium and Kinetic Studies on Reactive Dye Adsorption Using Palm Shell Powder (An Agrowaste) and Chitosan. J. Chem. Eng. Data.,v. 56, p. 35-42, 2011.

VERMA, A.K., DASH, R.R., BHUNIA, P.A.A review on chemical coagulation/flocculation technologies for removal of colour from textile wastewaters. J. Environ. Manage.,v. 93, p. 154$168,2012$.

WAN NGAH, W.S., TEONG, L.C., HANAFIAH, M.A.K.M. Adsorption of dyes and heavy metal ions by chitosan composites: A review. Carbohydr. Polym.,v. 83, p. 1446-1456, 2011.

WESKA, R.F., MOURA, J.M., BATISTA, L.M., RIZZI, J., PINTO, L.A.A. Optimization of deacetylation in the production of chitosan from shrimp wastes: use of response surface methodology. J. Food Eng.,v. 80, p. 749-753, 2007.

ZHANG, H., NEAU, S.H. In vitro degradation of chitosan by a commercial enzyme preparation: Effect of molecular weight and degree of deacetylation. Biomaterials,v. 22, p. 1653-1658, 2001. 